\title{
Revisión sobre el aceite de oliva: historia, impacto mediático y sus aplicaciones médicas en la dieta mediterránea
}

\author{
Review on olive oil: history, media impact and its medical implications \\ in the mediterranean diet)
}

\section{Rafael Gómez-Cuevas}

${ }^{*}$ Médico Univ. Complutense de Madrid. ExVicepdte. World Obesity Federation WOF . ExPdte. Federación Latinoamericana de Obesidad FLASO. ExPdte ACE, ASCOMTA. Prof. Emérito Hosp. Samaritana. Director Académico FECCOM, European Fellow Specialist Obesity I.A.S.O.

Fecha de recepción: 16/05/2018

Fecha de aceptación: 27/08/2018

\section{Resumen}

$\mathrm{E}$ aceite de oliva es definitivamente un "líquido de oro". La presencia del olivo en nuestra civilización se ha convertido en un símbolo auténtico. Aparece con el amanecer de nuestra historia: Desde la rama de olivo que trajo la paloma al arca de Noé, su valor como un premio olímpico, su imagen como un signo de paz y su acendrada presencia en los cultos religiosos, el olivo y el aceite de oliva han sido protagonistas de todos los siglos de nuestra memoria. A su sombra se ha desarrollado casi toda nuestra cultura. Ha sido cantado por poetas y también vanagloriado. Ahora su valor se acrecienta al convertirse en un verdadero aliado de la medicina. Como si estuviese cumpliendo un precepto de Hipócrates, hoy sabemos que el aceite del bendito olivo puede ayudar a prolongar nuestras vidas. Convertido en el pilar fundamental de la dieta mediterránea se yergue como un bastión en la prevención del infarto de miocardio. Este, nuestro aporte, pretende sucintamente recrear trozos de la historia, figuración literaria, simbolismo, y de la renovada valoración del aceite de oliva en el campo médico terapéutico y preventivo. Los médicos podemos afirmar hoy que el aceite de oliva tiene un bien ganado lugar en la prevención de las enfermedades cardiacas. Por eso, los herederos de Esculapio deberíamos recordarlo y valorarlo.

Palabras clave: Dieta mediterránea, ácidos grasos monoinsaturados, colesterol protector, símbolo de paz, premio olímpico, infarto de miocardio, aceite de oliva extra virgen.

\section{Summary}

Olive oil is definitely a "liquid gold". The presence of the olive in our civilization has become a symbol itself. It appears with the dawn of our history: From the olive branch that the dove brought to Noah's Ark, to its value as an Olympic prize, his image as a sign of peace and unblemished presence in religious cults, olives and oil olive have been protagonists of all our memories since then. Beneath its shadow most of our culture has flourished. It has been sung by poets and also worshipped Now its value is enhanced to become a true ally of medicine. As if it would be meant to fulfill a precept of Hippocrates now know that the blessed olive oil may help prolong our lives. Given the roll of main pillar of the Mediterranean diet stands as stronghold in the prevention of myocardial infarction. This, our contribution, aims to briefly recreate pieces of history, literary figuration, symbolism and renewed appreciation of olive oil in the therapeutic and preventive medical field. Doctors can say today that olive oil has a wellearned place in the prevention of heart disease. So - the heirs of Aesculapius - we should also take in the heart.

Key words: Mediterranean diet, monounsaturated fatty acids, protective cholesterol, peace symbol, olympic prize, myocardial infarction, extra virgin olive oil.

Objetivos: Al igual que -iniciado el proceso de elaboración del aceite de oliva - se habla de las bondades del dorado líquido de la primera extracción, pretendemos ahora exprimir las "aceitunas" de la literatura, reseñando lo claramente destacable, a nuestro juicio, que sobre el aceite y los olivares se ha escrito. El contenido es de verdad heterogéneo. También apasionante.

Les proponemos un viaje recalando en la historia, que se extiende anchurosa desde los griegos, fenicios y romanos hasta nuestros días, y que no pierde vigencia. Repasaremos su entronización como símbolo, y haremos un recuento bibliográfico de las palabras que ha merecido en poesía.. Finalmente, nos detendremos en lo que consideramos es digno de especial 
mención: el benefactor papel del aceite de oliva como preventivo del infarto, que los médicos valoramos tanto. Nos gustaría que al final todos estuviésemos de acuerdo en que el aceite de oliva es el protagonista de la ¡bendita sea! ¡Dieta mediterránea!

Echemos a andar.

\section{De su historia}

"Estamos en la segunda semana de noviembre, en pleno envero, cuando las olivas pierden su color verde tornándose moradas, casi negras".

Y podríamos iniciar este relato con la manida y un poco cursi frase ${ }^{(1)}$ de "El origen del olivo se pierde en la noche de los tiempos". Consignemos mejor algo ${ }^{(2)}$ con más "solera": algunos pasajes de la Biblia ${ }^{(3)}$. "El día veintisiete del séptimo mes se asentó el Arca... La paloma vino al atardecer, y he aquí que traía en el pico un ramo verde de olivo, por donde conoció Noé que habían disminuido las aguas, de encima de la tierra".

Si leemos el salmo 128:3: "Tu mujer será como vid que lleva fruto a los lados de tu casa; tus hijos como plantas de olivo alrededor de tu mesa".

Y en Éxodo, 3022-33: "El Señor dijo a Moisés: Toma perfumes de gran precio, cinco kilos de mirra en grano, y tres litros y medio de aceite de oliva. Con estos ingredientes harás el aceite de la unción santa, ungirás a Aarón y sus hijos, y los consagrarás para que estén a mi servicio como sacerdotes. Este será el aceite en todas vuestras generaciones: El que lo derrame sobre un laico, será excluido de su pueblo".

Recordemos también ${ }^{(3)}$ que cuando Jesús de Nazaret entró en Jerusalén el pueblo lo recibió con ramas de olivo. Significaba un homenaje. Y en la narración "en vivo" del Calvario aclaran que el Huerto de los Olivos en lengua hebrea se llamaba entonces Getsemaní, que significa "prensa de aceite"(4). Sigue presente un árbol de olivo, ya que Judas arrepentido se ahorca luego en una de sus ramas. Otro drama.

En el Corán ${ }^{(5,6)}$ también es señalado: "Dios es la luz de los cielos y la tierra. Su luz es como la de un candil en una hornacina... se enciende gracias al árbol bendito del olivo, el árbol que no es oriental ni occidental, cuyo aceite alumbra casi sin tocar el fuego: es luz de la luz".

Desde Egipto: "Han encontrado un ramo de olivo en la tumba de Tutankhamon".

Desde $^{(7)}$ la recién nombrada Atenas: "Conflicto sociopolítico con Poseidón; La diosa Palas, al hundir su lanza en tierra griega hizo que naciera el olivo. Se convirtió en símbolo de la vida y de la paz. Sentencia Zeus. Vencido Poseidón, Gana el olivo".

Y desde la sede de los juegos olímpicos celebrados en el año 776 a. C.: "Los ganadores recibieron como premio una rama de olivo", aunque luego algún generoso patrocinador agregara el regalo de ánforas de aceite, las panatenaicas ${ }^{(8)}$. Siglos después — homenaje de recuerdo - en los Juegos Olímpicos de 1896 y de 2004 también fueron premiados los ganadores con sendas ramas de olivo". Respetar la tradición.

Podemos resumirlo: griegos, fenicios, romanos, judíos, cartagineses, árabes e hispanos ${ }^{(9)}$ hicieron del olivo mediterráneo un símbolo de paz, cultura y vida.

\section{De su entronización como símbolo}

"Para recoger la aceituna tendemos la berraza y vareamos".

El olivo reúne una gran riqueza simbólica ${ }^{(10)}$ reconocida desde hace siglos. Se le atribuyen como virtudes propias: "Inmortalidad: porque vive, da fruto, y se renueva desde hace miles de años. Esperanza: Después de que Jertes incendiara la Acrópolis, solo el olivo sagrado del templo había crecido un codo en la primera noche, como imagen de renovación. Sabiduría: al tomar los atributos de la diosa Atenea. Fuerza: la maza de Hércules era de madera de olivo y de ella salían raíces, que se convertían en árbol, cuando se clavaba en el suelo. Fertilidad: para los helenos, los descendientes de los dioses nacían bajo los olivos, por lo que las mujeres que querían engendrar, dormían bajo su sombra. Victoria: Atenea le otorga este atributo al salir victoriosa de su lucha con Poseidón".

Una rama de olivo es símbolo mundial de la paz. Sus ramas aparecen en los emblemas de muchos países. En el pabellón de $\mathrm{ONU}$, la corona de ramas de olivo en todo el mundo simboliza la paz universal. Ojalá algún día la respeten de verdad.

Se nos ha encomendado que hilvanemos unas palabras sobre el aceite de oliva en su relación con la Medicina. De hecho, es el tema principal de esta revisión. Pero no pudimos resistir la tentación de entresacar — solo para los interesados-una reseña bibliográfica sobre el olivo y la poesía ${ }^{(11-16)}$. Y recordar que lo han cantado poetas como Miguel Hernández ${ }^{(17)}$, Machado $^{(18)}$, Alberti $^{(19)}$, Zorrilla(20), Neruda ${ }^{(21)}$ y García Lorca ${ }^{(22)}$.

\section{De la Medicina}

"Recogido ya el aceite de la 'primera prensa', estamos ante el panel de cata. Se va a escoger el mejor aceite de oliva 'Virgen Extra' (AOV)".

Su relación con la Medicina viene de lejos: en Babilonia nos llamaban a los médicos "asu" es decir especialistas en... aceites.

En Grecia, el padre de la Medicina, Hipócrates, sentenciaba: "Que la comida sea tu alimento y el alimento tu medicina"(23). Es el resumen estricto de los últimos cinco mil libros que sobre la obesidad se han escrito.

Nuestro "patrón" definitivamente tenía buen gusto cuando afirmaba sin sonrojarse que "El vino es una cosa maravillosamente apropiada para el hombre si, en tanto en la salud como en la enfermedad, se administra con tino y justa medida". Y para que no se nos olvidara lo dejó escrito en una "Biblia" médica: el Corpus Hippocraticum. 
El aceite de oliva lo recomendaba Dioscórides para tratar los problemas musculares. El cronista de Pompeya, Plinio el viejo, recopila las recetas conocidas a base de aceite de oliva. Escribe su obra Tacuinum sanitatis. Un correcto consejo dietético y nutricional.

Si nos situásemos en uno de los mercados de Roma, el jefe de cocina que se acercaba a comprar unas ánforas de aceite, preguntaría por el Óleum Baeticae (importado desde Sevilla). Elegiría entre distintas categorías perfectamente etiquetadas y marcadas con la procedencia y los sellos aduaneros preceptivos: - Óleum ex albis ulivis, de olivas verdes, amargo pero considerado de gran calidad. El mejor era el Oleum Primae pressurae. Ya entonces se valoraba, como lo hacemos nosotros, el aceite de la "primera prensa". Durante siglos, los felices pobladores de las riveras del Mediterráneo ${ }^{(24,25)}$ vivían muy bien, muchos más años, apenas sin infartos. Su "trilogía alimentaria"(26) estaba constituida por el olivo, el trigo y la vid. Solo hasta hace poco se comprobó que la "culpa" de ese bienestar la tenía el AOV. Esto permitió hacer separaciones, más bien "divorcios" en la familia de lípidos. Un consejo de guerra científico "bendijo" al HDL como protector. Y relegó a las tinieblas de malignidad a otra fracción hecha de partículas diminutas a mal colesterol. Parece ser, que sí es malo de verdad, que mata y por eso se llama LDL.

Keys $^{(27)}$, aunque no era médico, hizo la promoción con su trabajo “Sobre los siete países." En 2010, la UNESCO incluyó la dieta mediterránea (Dmed) en la lista del Patrimonio Cultural Inmaterial.

Cabe preguntar aquí, ¿quién sería el "presunto" culpable de las bondades adscritas al AOV? Tenemos rápida respuesta: Es el ácido oleico, cuya "traducción" bioquímica es ácido graso monoinsaturado de la serie omega. Son aceites vegetales como de oliva, aguacate, etc. que reducen el riesgo de ECV. El ácido oleico se encuentra en la mayoría de las grasas y aceites naturales ${ }^{(28)}$.

El AOV posee un alto índice de ácidos grasos monoinsaturados antioxidantes. Contiene: ácido oleico 73\%, palmítico $12 \%$, esteárico $4 \%$, linoleico $8 \%$ y linolénico 1,2\%.

Tanto el oleico (monoinsaturado) como el linoleico (poliinsaturado) son esenciales y nuestro organismo no puede sintetizarlos. Son VIP. Pero están en nuestra comida diaria. Por ello urge para Latinoamérica una reflexión en nuestros sistemas de alimentación como denuncia Popkins ${ }^{(29)}$ en su completísimo y reciente trabajo de abril de 2018.

Preguntan, ¿sirve el aceite de oliva "simple" o va mejor el "virgen extra"? He aquí la respuesta con ribetes científicos: El AOV se obtiene a partir de la primera prensada de las aceitunas y tiene un específico y característico elevado contenido en antioxidantes ${ }^{(28,30)}$. Esto es esencial. Es justamente lo que lo distingue: aumenta la capacidad antioxidante del plasma y la resistencia a la oxidación del LDL. Mejor elección el AOV.

Siempre insistimos - previendo factores de riesgo frente a un paciente - que no solo somos "lipidólogos" — vigilar niveles de glucosa y ácido úrico. Es absolutamente necesario vigilar el peso y prevenir la obesidad ${ }^{(30,31,34)}$.

La mayoría de los factores de riesgo de cardiopatía coronaria operan en ambos sexos. Pero la mujer tiene un panorama metabólico más amable: su colesterol HDL protector es alto, aunque debe vigilar sus niveles de glucosa y triglicéridos.

Pero en resumen y porque ya es hora de aliñar una ensalada con el extra virgen que hemos logrado extraer, deben aflorar también algunas conclusiones: El efecto beneficioso del AOV en el riesgo de la cardiopatía se debe en primer lugar a sus efectos favorables en los lípidos sanguíneos, incluyendo su oxidación, y luego a la protección de la funcionalidad endotelial, base fundamental para prevenir las enfermedades cardiovasculares.

En otras palabras: sin pecar de optimistas, podemos sugerir que la alimentación ${ }^{(35)}$ previa con dieta rica en AOV protege, reduciendo el daño miocárdico y vascular, además de formación de trombos, "haciendo que la intensidad, en su conjunto, de estos daños sobre el corazón sea menor.” Estupenda noticia.

¿Pero tenemos ya evidencias claras, científicas, como nos gusta ahora a los médicos, que sean "basadas en la evidencia”? ¿Debidamente contrastadas con datos clínicos que lo confirmen? Y he aquí que encontramos esta noticia: "Beneficios de la dieta mediterránea. Estudio PREDIMED ${ }^{(32)}$ en New England Journal of Medicine del 25 de febrero de 2013, cuyo resumen dice: "PREDIMED (Prevención con Dieta Mediterránea) es un ensayo clínico aleatorizado de intervención dietética que pretende averiguar si la dieta mediterránea (Dmed) suplementada con (AOV) o frutos secos evita la aparición de complicaciones cardiovasculares mayores (muerte de origen cardiovascular, infarto de miocardio y/o accidente vascular cerebral) en sujetos de alto riesgo vascular, comparado con una dieta baja en grasa.

En este ensayo, la Dmed suplementada con AOV logra una reducción del riesgo relativo del $30 \%$ entre las personas de alto riesgo. La magnitud de este beneficio es impresionante, una intervención dietética, con resultados verdaderamente notables y apoyan los beneficios de la Dmed para la reducción del riesgo cardiovascular. Los resultados ${ }^{(32,33)}$ refuerzan su valor a nivel internacional.

¿Cuáles fueron sus objetivos?: Valorar los efectos de la Dmed - suplementada con AOV_ - sobre la incidencia de complicaciones cardiovasculares mayores como infarto de miocardio. También se valoraron la adición de frutos secos y la ingesta moderada de vino. De los 7.447 participantes, un total de 2.543 recibieron AOV a razón de 1 litro semanal.

\section{¿Cómo la investigación médica ha llegado hasta aquí?}

Así como el más largo camino precisa siempre un primer paso, los estudios y trabajos previos merecen ser considera- 
dos: En 1948, Ancel Keys "predijo" en un artículo publicado en la revista holandesa Voeding que las enfermedades del fin de siglo estarían relacionadas con los excesos dietéticos y la arteriosclerosis. Keys estuvo en España en 1951, invitado por el profesor Jiménez Díaz. Recién llegado se reunió con Grande Covián e iniciaron un estudio de niveles de colesterol en madrileños de distintos barrios. Los primeros resultados marcaron el rumbo de sus investigaciones. ¿Qué había llamado su atención? Pues que - como cuenta en sus memorias- los habitantes de barrios obreros (Vallecas y Cuatro Caminos), que apenas bebían leche ni comían mantequilla o carne, tenían valores muy bajos y las enfermedades coronarias eran prácticamente desconocidas. Ese dato contrastaba con lo hallado en los bien acomodados habitantes del barrio Salamanca, que "gozaban" de una dieta mucho más rica en grasas de origen animal, valores de colesterol más elevados y "padecían" más infartos de miocardio. Creemos que esta observación sabiamente valorada por Key, es el punto de partida para realizar el primer estudio epidemiológico multinacional sobre la dieta, colesterol y enfermedad coronaria, conocido como "Estudio de los Siete Países". Su hipótesis dieta-lípidos-corazón lo hizo famoso y motivó su aparición en la portada del Time Magazine como "Mr. Cholesterol". Se jactaba de conocer muy bien a España y su gastronomía, así que publicó, junto con Margaret, su esposa, varios libros en los que pregonaban las ventajas de la “dieta mediterránea” (Dmed), término acuñado por Keys en su laboratorio de Minnesota.

La Dmed, "presentada" por primera vez en ese estudio, es considerada $^{(35)}$ el patrón de oro para una nutrición saludable y asociada con reducción de la morbimortalidad de tipo cardiovascular. Se dijo entonces que salvaba vidas, ¿las sigue salvando? Claro que sí( ${ }^{(36)}$.

La Dmed es baja en grasas saturadas, rica en legumbres, fibra, vitaminas antioxidantes y AOV como principal fuente de grasa.

El "abogado del diablo" preguntaría con toda razón, si hay o hubo estudios comparativos. Lo revisamos: En el North Karelia Project ${ }^{(37)}$ se buscaba valorar si era beneficioso recomendar cambios en la dieta de un grupo poblacional. Tras un adecuado consejo nutricional se logró una valorable reducción de mortalidad vascular en hombres adultos. Impresionante. Como dato anecdótico se recuerda que "entre 1982 y 2007 el porcentaje de energía aportada por el consumo de grasas en Finlandia bajó del $40 \%$ al $25 \%$. La clave: empezar a utilizar aceites vegetales. Y "bajar también el consumo de mantequilla de $18 \mathrm{Kg}$ al año por habitante a $3 \mathrm{Kg}$ en 2005”. Inapelable.

\section{¿Y cómo se aplica esto en la práctica?}

Siguiendo a la Sociedad Española de Arterioesclerosis, Medina señala ${ }^{(38)}$ en el apartado de Ingesta adecuada de grasas: "Un aporte con proporción adecuada de grasa es preciso para lograr una dieta equilibrada. Las recomendaciones pasan obligatoriamente por incluir el AOV en todas sus variedades y posibilidades culinarias". Parece una sugerencia valorable. Fácil. Práctica.

En los consejos sobre dieta para la enfermedad coronaria $^{(39)}$ la SEEN (Sociedad Española de Endocrinología y Nutrición) insiste en que "Los ácidos grasos monoinsaturados aportarán hasta el 15\% de las calorías totales. El AOV y los frutos secos son ricos en estos ácidos grasos. Es saludable incorporar este aceite en la alimentación habitual en remplazo de los ácidos grasos saturados". Suena convincente.

Las recomendaciones de la Sociedad Española de Dietética ${ }^{(40)}$ aconsejan "mantener el consumo de grasa saturada en $<10 \%$ y el de trans en $<1 \%$ de la ingesta calórica y seguir un patrón de Dmed, ya que ayuda a prevenir numerosas enfermedades crónicas, típicas de nuestro tiempo". Esto ya parece contundente.

En el libro ${ }^{(41)}$ Consejos para personas que han superado una angina o un infarto, una segunda oportunidad, en el capítulo sobre Factores de Prevención, textualmente se afirma: "Cocine y aliñe los alimentos con AOV” Es como una admonición.

No lo estamos amenazando, refrescamos su memoria: "Las enfermedades cardiovasculares son la principal causa de morbimortalidad en Europa , si no se modifica el estilo de vida, se puede esperar un aumento del número de muertes en los próximos años. La adopción de una Dmed ${ }^{(42)}$ en la que el AOV es la principal fuente de grasa es una excelente forma de mejorar el factor "nutrición" implicado en la prevención de las enfermedades cardiovasculares. "Es mejor prevenir que curar".

Y para contestar, ¿cuál es la dieta óptima? Seedorf y col ${ }^{(43)}$ nos dan este consejo: "Deben restringirse las grasas saturadas. En cambio, la tradicional Dmed, cuya principal fuente de grasas es el AOV, contiene sustancias antioxidantes, vitaminas E y C, así como polifenoles y juega un papel muy importante en la prevención de las enfermedades cardiovasculares". Más claro, más explícito, más resumido es imposible. Y, además, es verdad.

¿Pero se ha seguido investigando? Desde luego, ya está marchando el Estudio Predimed plus "Efecto de una Dmed con restricción de energía, actividad física y tratamiento conductual sobre la prevención de enfermedad cardiovascular. Abril de 2018"(44).

En este nuevo ensayo multicéntrico participan 23 centros reclutadores, incluyendo un total de 6.874 participantes, 3.406 asignados al grupo de intervención intensiva y 3.468 al control Dmed tradicional. Se trata de un patrón alimentario rico en grasas de fuentes vegetales naturales (AOV y frutos secos), bajo consumo de carnes rojas o procesadas, moderada ingesta de pescado y vino.

En el protocolo ${ }^{(44)}$ de este nuevo ensayo se valoran estos mecanismos de acción ${ }^{(45)}$ : reducción en la inflamación de bajo grado, mayores niveles de adiponectina, menor coagulabilidad, mejoría de la función endotelial, menor estrés oxidativo, 
menor concentración de lipoproteínas aterogénicas, menores niveles de partículas LDL oxidadas y menor captación por los macrófagos de las LDL oxidadas. Perfil de ácidos grasos saludable y contiene ${ }^{(48)}$ numerosos compuestos fenólicos bioactivos que tiene propiedades antiinflamatorias, impacto beneficioso sobre el perfil lipídico, mejoría de marcadores de estrés oxidativo, efecto antiagregante plaquetario y estimula la biogénesis mitocondrial. Explican el efecto antiaterogénico de la Dmed. En un subestudio también se observó reducción de 48\% el riesgo de incidencia de DM2.

Respecto a datos estadísticos: Comparando la Dmed con otras respecto a sus efectos sobre la salud en adultos se hizo un estudio con las fuentes: Ovid, MEDLINE, CINAHL, y the Cochrane Library desde 1990 hasta abril de 2016 (46). La conclusión de Bloomfield: "Puede sugerirse que una Dmed sin restricciones en la ingesta de grasa puede reducir la incidencia de eventos cardiovasculares y DM2."

Estudios epidemiológicos prospectivos respaldan los beneficios del patrón Dmed para reducir el riesgo de enfermedades crónicas y mejorar la calidad de vida. Evidencia relevante ${ }^{(47)}$ de los efectos de la Dmed y el estilo de vida en la salud (2015/2016). "Recientemente, 19 nuevos estudios prospectivos mostraron, con una consistencia casi perfecta, fuertes beneficios de Dmed para reducir el riesgo de infarto de miocardio, accidente cerebrovascular, mortalidad total e insuficiencia cardiaca".

Papel de los polifenoles ${ }^{(48)}$ : "Están demostradas las propiedades antiinflamatorias, antioxidantes y antiangiogénicas de los polifenoles del AOV; sin embargo, se desconocen los efectos de la fracción fenólica del aceite de oliva sobre la angiogénesis inflamatoria. En este estudio, Calabriso investiga los efectos del extracto polifenólico de aceite de oliva, y metabolitos circulantes relacionados con las respuestas angiogénicas inducidas por VEGF y la actividad y expresión de NADPH oxidasa en células endoteliales humanas cultivadas. Sugieren que: Los polifenoles nativos y sus metabolitos disminuyen las respuestas angiogénicas inducidas por VEGF al prevenir la actividad endotelial de NADPH oxidasa y disminuir la expresión de las subunidades selectivas de NADPH oxidasa".

Papel de la dieta mediterránea en diabéticos ${ }^{(49)}$ : La evidencia científica sobre los beneficios de la Dmed, dice VidalPeracho, respalda la recomendación de aumentar su consumo en DM2 y promoverla entre los más jóvenes de la muestra estudiada.

Los cardiólogos también preconizan la Dmed como factor de prevención de la enfermedad coronaria(50): "La primera causa de morbilidad y mortalidad en el siglo XXI continúa siendo la ECV. Según las previsiones de Arós F. y Estruch R, la situación no variará en las próximas décadas. La baja incidencia de morbimortalidad por enfermedad coronaria en los países mediterráneos despierta un gran interés. El número de publicaciones en PubMed sobre este tema ha aumentado de menos de 10 en 1985 a cerca de 300 en 2012: un incremento en la adherencia a la Dmed se acompaña de una reducción del riesgo vascular.

También en Latinoamérica ${ }^{(51)}$ Dussaillant describe buenos resultados: "El estilo Dmed ha demostrado, con amplia solvencia científica, generar numerosos beneficios en la prevención y tratamiento de diferentes patologías crónicas. La evidencia revisada sugiere que la alimentación Dmed es una importante herramienta para disminuir la morbimortalidad prematura en la población chilena".

Sobre efectos a largo plazo(52): Sacanella confirma que "Los participantes de Dmed tuvieron concentraciones celulares y plasmáticas más bajas de marcadores inflamatorios relacionados con la aterosclerosis a los 3 y 5 años".

Como somera conclusión: la Dmed explica su efecto cardioprotector a largo plazo, gracias a los comprobados beneficios del AOV. No dudamos de buena fe en aconsejarla. Parece plausible sugerir que utilicemos esta valiosa herramienta terapéutica en nuestro próximo paciente. 


\section{Referencias}

1. "El origen del olivo". Consultado en 4/03/2017 en www.mondoliva.com

2. Historia el aceite de oliva. Consultado en 4/03/2017 en www.aceitedeoliva.com

3. Esencia del olivo. "Historia del olivo" consultado 14/03/2016 www.esenciadeolivo.es

4. Picornell Buendía, M.R. Melero Martínez, J.M: "Historia del cultivo del olivo y del aceite; su expresión en la Biblia”, en Ensayos Revista Facultad Educa ción de Albacete, N. 을 28,2013., (155-181) (Enlace web: http://www.revista. uclm.es/index.php/ensayos

5. El olivo, un árbol mítico, símbolo de la paz. Consultado 5/5/2015 en paginasarabes.com

6. El olivo. Coran.An-hour consultado el 05/5/2015 en www.islamicbulletin. org/newsletter/issue25spanish/oliveoil.aspx

7. Historia del aceite de oliva y su influencia en la cultura. Tomado el 02.04.2015 de https://www.aceitedeoliva.com/aceite-de-oliva/historiadel-aceite-de-oliva/

8. Historia del aceite de oliva en la cultura mediterránea. Por qué el olivo es el árbol del mediterráneo. Esencia del olivo En:ibericodirecto.com/historiadel-aceite-de-oliva/ Consultado en octubre de 2017.

9. Bolens Lucie "Riquezas de la tierra andaluza y primacía del aceite de oliva en la sociedad y la civilización del Al Andalus". En: Agricultura y Sociedad 1996 N 80-81 pp 181-216

10. Simbología del olivo. Extracto de Libro del aceite y la aceituna March L Rios A. Consultado 25/03.2017 de www.museodelaceite.com

11. El Olivo como símbolo de Paz | Ruta del Olivo https://rutadelolivovila.wordpress.com consultado marzo 2018

12. Rincón García Wifredo "El olivo en el arte español" 2005. Jaén. I Congreso Cultura del Olivo.

13. Poemas del Olivar - www.juntadeandalucia.es/averroes/lamagdalena/poemas_olivar.htm

14. El olivo y el aceite de oliva en la poesía - Ministerio de Agricultura, Alimentación y Medio Ambiente. En www.magrama.gob.es/ministerio/pags/exposiciones/poesia 01.html consultado Novbre. 2017

15. Gómez Pérez A. "El flamenco del olivar en la poesía popular"408-444 tomado de: institucional.us.es/revistas/rasbl/38/art_29.pdf

16. Santano Antonio El olivar de la luna: El olivo en la poesía. En: www.elolivardelaluna.com/2008/02/el-olivo-en-la-poesia.htm

17. Hernández Miguel Obra completa Espasa libros, 2010 ISBN 9788467032628

18. Antonio Machado, el poeta del olivar, en www.aledooleo.es/antonio-machado-el-poeta-del-olivar

19. Rafael Alberti - Poemas - Mundo Latino en www.mundolatino.org/cultura/ alberti5.htm

20. Don Juan Tenorio en www.juntadeandalucia.es/averroes/iestorredelosherberos

21. Pablo Neruda en www.olivasnaturales.com.ar/poesiayolivo.htm

22. Romancero Gitano - Los Poetas en www.los-poetas.com/a/lorca1.htm

23. Tratados Hipocráticos I. Madrid, Editorial Gredos, 1990, pp. 399-400.]

24. Lillo Carpio Pedro A. "El aceite en el Mediterráneo antiguo" Revista Murciana de Antropología 2000 N7pag 57-75.

25. Grande Covián F. El aceite de oliva en la prevención de enfermedades cardiovasculares. Ponencia II. Simposio Científico-Técnico. 1985 Expolia. Jaén

26. Zugasti Murillo Ana: La dieta mediterránea patrimonio inmaterial de la Unesco. en fmed.org/dieta-mediterránea, enero 2012, Endocrinología.

27. Keys Ancel y Keys Margaret. Eat well and stay well. Double Day. New York. Reseñado JAMA ,1963,184 ISBN: 0385065752.

28. Zamora Ardoy MA, Báñez Sánchez F, et al. Aceite de oliva: influencia y beneficios sobre algunas patologías. An Med Interna (Madrid) 2004; 21: 138142

29. Popkin B.M. Reardon T. Obesity and the food system transformation in Latin America Obesity reviews Abr 2018 onlinelibrary.wiley.com Consultado 6 mayo, 2018.

30. Serra-Majem L, Roman B, Estruch R. Scientific evidence of interventions us- ing the Mediterranean diet: a systematic review. Nutr Rev 2006; 64: S27-47.

31. "Estrategia NAOS. Invertir la tendencia de la obesidad. Estrategia para la nutrición, actividad física y prevención de la obesidad". Madrid: 2005 Agencia Española de Seguridad Alimentaria. Ministerio de Sanidad y Consumo.

32. Estruch R., Ros E, "Primary Prevention of Cardiovascular Disease with a Mediterranean Diet PREDIMED Study Investigators." N Engl J Med 2013; 368:1279-1290.

33. “El estudio PREDIMED sobre dieta mediterránea, entre los más relevantes para 'The NEJM Journal Watch General Medicine' En : www.ciberobn.es/index consultado Octubre 2017.

34. Gómez-Cuevas R. "Aspectos clínicos de la Obesidad." Del libro Obesidad una autopista hacia el infarto 1984 Gráficas Carman Pag. 15-21.

35. Keys et al. "The diet and 15 year death rate in the Seven Countries Study. American Journal Epidemiology 1986, 124,903-915.

36. Siani a, Ferrara A. Mediterrean Diet Nutritional and Metabolic bases of Cardiovascular diseases, Mann J. Chap15,2011 edited Mario Mancini.

37. Vartiainen E. Laatikainen T. el at. Thirty-five-year trends in Cardiovascular Risk Factors in Finland Int J. Epidemiology 2010; 39, 504-18.

38. Medina Francisco Javier coord. "La alimentación Mediterránea, Historia Cultura, tradiciones 1996 www.dialnet uniría consultado Novbre. 2017.

39. Sociedad Española de Dietética. Sociedad Española de Endocrinología y Nutrición en www.nutricion.org y http://www.seen.es/docs/apartados/1663/Las\%20grasas\%20en\%20nuestra\%20alimentaci\%C3\%B3n. pdf consultado Abril 2018.

40. Carrillo Fernández L Dalmau Serra J. "Grasas de la dieta y Salud Cardiovascular Clin Invest Arterioscl 2011,23 Supl1, 1-36.

41. De la Torre Prados et al. "Consejos para personas que han superado una an gina o un infarto "Edit. Junta de Andalucía .2011, Consejería de Salud.

42. Zamora Ardoy, M. A.; Banez Sanchez, F. et al. "Aceite de oliva: influencia y beneficios sobre algunas patologías" An. Med. Interna (Madrid) 2004, vol21, n 3 pag138-142.

43. Seedorf U, Schulte H Assmann G. "Genes, diet and public health" Genes Nutr.2007 Oct, $2,75-80$

44. Salas-Salvadó J Mena G., Nutr Clin Med 2017; XI (1): 1-8 DOI: 10.7400/NCM 2017.11.1.5046

45. Tomado de www.predimedplus.com/wp- content/uploads/- PREDIMED PLUS_consultado Abril_2018_03042018.pdf

46. Bloomfield HE, Koeller E, et al. Effects on Health Outcomes of a Mediterranean Diet With No Restriction on Fat Intake: A Systematic Review and Meta-analysis. Ann Intern Med. 2016 Oct 4;165(7):491-500.

47. Martinez-Gonzalez MA, Martin-Calvo N. Mediterranean diet and life expectancy; beyond olive oil, fruits, and vegetables. Curr Opin Clin Nutr Metab Care. 2016 Nov; 19 (6): 401-407.

48. Calabriso N,Massaro M. et al. Extra virgin olive oil rich in polyphenols modulates VEGF-induced angiogenic responses by preventing NADPH oxidase activity and expression The Journal of Nutritional Biochemistry, Volume 28, February 2016, Pages 19-29.

49. Vidal-Peracho, C. Tricás-Moreno, J.M. et al. Adherence to Mediter ránea Diet Pattern among Spanish Adults Attending a Medical Centre: Nondiabetic Subjects and Type 1 and 2 Diabetic Patients. Journal of Diabetes Research.Volume 2017, Article ID 5957821, 11 pages 50. Arós F. Estruch R. Dieta mediterránea y prevención de la enfermedad cardiovascular Rev Esp Cardiol. 2013;66:771-4 - Vol. 66 Núm.10 DOI: 10.1016/j.recesp.2013.04.026

51. Dussaillant C, Echeverría G, Urquiaga I, et al. Evidencia actual sobre los beneficios de la dieta mediterránea en salud. Rev Med Chile 2016; 144: 1044 1052.

52. Sacanella E, et al. Long-Term Immunomodulatory Effects of a Mediterranean Diet in Adults at High Risk of Cardiovascular Disease in the (PREDIMED) Randomized Controlled Trial. J Nutr. 2016 Sep;146(9):1684-93. 